\title{
FORMACIÓN DOCENTE PARA ATENDER A LA DIVERSIDAD. UNA EXPERIENCIA BASADA EN LAS TIC Y EL DISEÑO UNIVERSAL PARA EL APRENDIZAJE
}

\section{TEACHERS TRAINING TO DEAL TO DIVERSITY. AN EXPERIENCE BASED ON ICT y UNIVERSAL DESIGN FOR LEARNING}

Sergio Sánchez Fuentes ${ }^{1}$. Universidad Católica de Murcia. España. sergio.sanchezfuentes@uam.es

Rosario Ángela Martín Almaraz. C.E.E. Nuestra Señora de la Esperanza de Segovia. España.

rosariomartin@gmail.com

\section{RESUMEN}

Este trabajo aborda la importancia de la formación continua del profesorado en la atención a la diversidad en el aula. Para ello, se ha tenido en cuenta lo descrito por el paradigma del diseño universal para el aprendizaje (DUA) y su relación con el uso de las tecnologías de la información y la comunicación (TIC). El uso de las TIC se plantea como un avance en el acceso a la educación por parte de todos los estudiantes, sin embargo, también este uso puede suponer una barrera si no se tienen en cuenta los diferentes tipos de discapacidad que están presentes en las aulas. Este trabajo presenta una experiencia de formación centrada en aprender sobre la discapacidad y el uso de las TIC, describir el enfoque del DUA y avanzar en el conocimiento sobre accesibilidad en herramientas ofimáticas. Los principales resultados muestran que los participantes valoran muy positivamente la formación, que las competencias adquiridas suponen un avance en su formación y que tienen aplicabilidad en el aula. Como conclusión, se describe como la formación para atender a la diversidad debe centrarse en el uso de las TIC pensando en todos los estudiantes, siendo el DUA un medio óptimo para lograrlo.

PALABRAS CLAVE: diseño universal para el aprendizaje, formación, TIC, diversidad.

\section{ABSTRACT}

This paper addresses the importance of training teachers to respond to diversity in the classroom. With this objetive, the Universal Design for Learning (UDL) and the use of Information and Communication Technology (ICT) have been described. The progress in the use of ICT can to improve the access to education by all students, but

\footnotetext{
${ }^{1}$ Sergio Sánchez Fuentes: Profesor ayudante doctor en la Universidad Autónoma de Madrid en el Departamento de Psicología Evolutiva y de la Educación en la Facultad de Formación del profesorado. Doctorado en la Universidad de Salamanca. Ha trabajado como profesor universitario en la Universidad de Salamanca, en la Pontificia Universidad Católica del Ecuador, en la Universidad Católica de Murcia y en la Universidad de Burgos.
} 
it can also be a barrier if we have not taken into account the different types of disabilities that are present in classrooms. Thus, an experience carried out in teachers training courses is presented. A seminar focusing on dealing with disability and the use of ICT has been developed, describing the approach to universal design learning and learning about accessibility with some office tools. The main results show that participants value the training highly, the skills acquired are benefits in their training and that there is applicability of these competences in the classroom. In conclusion, this paper describes how training for dealing with diversity should focus on the responsible use of ICT, with the universal design for learning as an optimal means to achieve it.

KEY WORDS: universal design for learning, training, ICT, diversity.

\section{Cómo citar el artículo:}

Sánchez Fuentes S. y Martín Almaraz, R. A. (2016). Formación docente para atender a la diversidad. Una experiencia basada en las TIC y el diseño universal para el aprendizaje. Revista de Ciencias de la Comunicación e Información, 21(2), 35-44.

doi: http://doi.org/10.35742/rcci.2016.21(2).35-44

\section{INTRODUCCIÓN}

La formación continua de maestros y profesores debe ser una prioridad en las políticas educativas de un país. Los cursos de actualización a profesionales en activo, deben incluir los principales enfoques que favorecen la atención a la diversidad en las aulas actuales, incluyendo éstos el uso de las Tecnologías de la Información y la Comunicación (TIC). Siendo uno de los objetivos, más intensamente perseguidos por parte de la comunidad educativa, la consecución de la plena participación de la diversidad de alumnado que caracteriza las aulas de los distintos niveles educativos.

Como uno de los principales medio para lograr atender a la diversidad, se encuentra la denominada educación inclusiva (Ainscow, 2006; Armstrong y Moore, 2004; Booth y Ainscow, 2002; Dyson, Howes y Roberts, 2002). Por lo tanto, ante esta necesidad y siguiendo los objetivos de la educación inclusiva, uno de los principales retos de la formación continua a maestros y profesores, debe ser el la de disponer de currículos accesibles que contemplen la diversidad de estudiantes dentro de las aulas (Díez, et al., 2011). Y es que un currículum diseñado universalmente, está específicamente diseñado para atender a un conjunto diverso de estudiantes con un amplio rango de habilidades sensoriales, motorices, cognitivas, afectivas y lingüísticas (Hitchcock y Stahl, 2003).

Pero, no solo son currículos deben accesibles, sino que en la programación del acto educativo, incluso en aquel en el que las TIC están presentes como un medio para lograr el objetivo, se debe tener en cuenta una serie de premisas de acceso al uso de las tecnologías, ya que no todos los estudiantes tienen las mismas habilidades o capacidades a la hora de enfrentarse a las TIC en el aula. (Ribeiro y Sánchez, 
2013). De este modo, un estudiante con discapacidad auditiva no tendrá las mismas posibilidades de aprendizaje ante un vídeo si éste no está bien subtitulado y contiene la información necesaria para la compresión del mismo. Igualmente, un estudiante con discapacidad visual ve mermadas sus oportunidades educativas si el uso del libro de texto es la única manera en la que los profesores enseñan, ya que en la mayoría de los casos sus explicaciones y sus actividades van a estar directamente relacionadas con lo que en dicho libro se describe. Éstos, aunque puedan suponer ejemplos estereotipados de lo que sucede en el aula a un alumnos con discapacidad, no se aleja mucho de la realidad en la que se encuentras muchos estudiantes si los profesores no son conscientes de la necesidad de tener en cuenta a la diversidad y las características individuales de sus alumnos.

\subsection{Diseño Universal para el Aprendizaje y su relación con el uso de las TIC en la formación docente}

El uso de las TIC proporciona flexibilidad en muchos aspectos relacionados con el aprendizaje, y una de las vías para lograr dicha flexibilidad, es a través de la adopción de concepciones abiertas e inclusivas respecto al modo de diseñar los procesos educativo (Ribeiro y Sánchez, 2013). El denominado diseño universal o diseño para todos, con origen en la arquitectura y el diseño de productos y servicios, es uno de los aspectos claves en relación al incremento en la participación de la estudiantes en la educación (Díez et al., 2011; Duk y Murillo, 2013). Además, este enfoque "aplicado a la educación puede ser un nuevo paradigma que permita hacer efectiva la implementación de la inclusión y proporcionar acceso a la educación general del currículo" (McGuire, Scott y Shaw, 2006. p. 167). Y es que, el diseño universal aplicado a la educación, puede suponer un paso más allá de la educación inclusiva. No solo es modificar los objetivos y contenidos de los currículos, a través de las denominadas adaptaciones curriculares, sino más bien diseñar los currículum desde el principio para que tengan la máxima variabilidad posible en los métodos de enseñanza y aprendizaje. De este modo, se pretende lograr que si el currículo está diseñando universalmente, no sean necesarias esas adaptaciones curriculares, o en el caso de necesitar realizarlas, éstas sean mínimas.

A pesar de que la aparición del diseño universal se produjo hace aproximadamente cuatro décadas, su repercusión y aplicación en el ámbito educativo se puede considerar como algo novedoso. Especialmente en los países de América del Norte y Japón este paradigma ha conseguido estar presente en las políticas educativas en la última década, ayudando este hecho a su difusión y su aplicación en el proceso de enseñanza.

Basándose en un estudio presentado por Ruiz, Solé, Echeita, Sala, y Datsira (2012), donde se describe el desarrollo del concepto del diseño universal aplicado en la enseñanza, se ha tomado en cuenta, por la envergadura, repercusión y disposición de trabajos y estudios que avalan su aplicación, lo que en la actualidad se conoce como Diseño Universal para el Aprendizaje (DUA). Y es que según la reciente publicación de Duk y Murillo (2013), el diseño universal para el aprendizaje "se sustenta en la experiencia del diseño arquitectónico, en los avances en el uso de las TIC en educación y en los hallazgos de investigaciones en neurociencias" (p. 12). 
EI DUA significa un nuevo marco para el currículum que permite realizar mejoras en el acceso a la educación por parte de todos los estudiantes, y especialmente de los estudiantes con discapacidad (Rose, Meyer y Hitchcock, 2005). EI DUA ayuda a tener en cuenta la variabilidad de los estudiantes proporcionando flexibilidad en objetivos, contenidos, materiales y evaluación. EI DUA se apoya en tres principios fundamentales que sirven como base para el desarrollo y transformación de los currículos. Estos tres principios, se derivan de numerosos estudios empíricos y en especial de investigaciones del ámbito de las neurociencias (Meyer y Rose, 2009; Rose y Meyer, 2000, 2002, 2006). Estos tres principios se pueden observar en la siguiente tabla.

Tabla 1. Principios y Pautas del Diseño Universal para el Aprendizaje (adaptado de NCUDL, 2012).

\begin{tabular}{|c|c|c|c|}
\hline & $\begin{array}{l}\text { Principio I. Proporcionar múltiples } \\
\text { formas de Representación } \\
\text { (el "qué" del aprendizaje) }\end{array}$ & $\begin{array}{l}\text { Principio II. } \\
\text { Proporcionar } \\
\text { Múltiples Formas de } \\
\text { Acción y Expresión } \\
\text { (el "cómo" del } \\
\text { aprendizaje) }\end{array}$ & $\begin{array}{l}\text { Principio III. } \\
\text { Proporcionar } \\
\text { múltiples Formas de } \\
\text { participación* } \\
\text { (el "por qué" del } \\
\text { aprendizaje) }\end{array}$ \\
\hline \multirow{3}{*}{$\stackrel{\infty}{\frac{\infty}{5}}$} & $\begin{array}{l}\text { Proporcionar opciones para la } \\
\text { percepción }\end{array}$ & $\begin{array}{l}\text { Proporcionar opciones } \\
\text { para la interacción } \\
\text { física }\end{array}$ & $\begin{array}{l}\text { Proporcionar opciones } \\
\text { para captar el interés }\end{array}$ \\
\hline & $\begin{array}{c}\text { Proporcionar múltiples opciones para } \\
\text { el lenguaje, los símbolos y las } \\
\text { expresiones matemáticas }\end{array}$ & $\begin{array}{l}\text { Proporcionar opciones } \\
\text { para la expresión y la } \\
\text { comunicación }\end{array}$ & $\begin{array}{l}\text { Proporcionar opciones } \\
\text { para mantener el } \\
\text { esfuerzo y la } \\
\text { persistencia }\end{array}$ \\
\hline & $\begin{array}{c}\text { Proporcionar opciones para la } \\
\text { comprensión }\end{array}$ & $\begin{array}{l}\text { Proporcionar opciones } \\
\text { para las funciones } \\
\text { ejecutivas }\end{array}$ & $\begin{array}{l}\text { Proporcionar opciones } \\
\text { para la auto-regulación }\end{array}$ \\
\hline
\end{tabular}

Diferentes estudios han demostrado que el entrenamiento eficaz de profesores, tanto de enseñanzas básicas como de enseñanzas superiores, es beneficioso para la creación de un currículum integrador para los estudiantes. Spooner, Baker, Harris, AhlgrimDelzell y Browder (2007), muestran que la formación del profesorado sobre cómo desarrollar currículo basado en el diseño universal para el aprendizaje, incluso realizada con acciones formativas breves en términos de tiempo, favorece el desarrollo de estrategias inclusivas en la creación de contenidos de aprendizaje haciéndolos más accesibles para todos los estudiantes. Engleman y Schmidt (2007) presentan un trabajo sobre la formación on-line siguiendo los principios del DUA con 216 participantes, en el que demuestran que los estudiantes prefieren los cursos desarrollados bajos estos principios, afirmando que la variedad de opciones que se derivan de la aplicación del DUA y la posibilidad de adecuarlas al propio estilo de aprendizaje, es percibida por los alumnos como un ambiente educativo que favorece el aprendizaje.

Una herramienta que demuestra la aplicabilidad del paradigma del DUA en la educación, fundamentalmente en la educación primaria y secundaria, son las denominadas Pautas 2.0 para el Diseño Universal para el Aprendizaje (NCUDL, 2012). Éstas son la base en la que a través de sus tres principios, concretados en nueve pautas, se ejemplifica como poder llevar acabo un diseño curricular siguiendo los parámetros propuestos por el DUA. De este modo, en la siguiente figura se pueden observar dichas pautas con sus respectivas relaciones entre principios. 


\section{Pautas de Diseño Universal para el Aprendizaje}

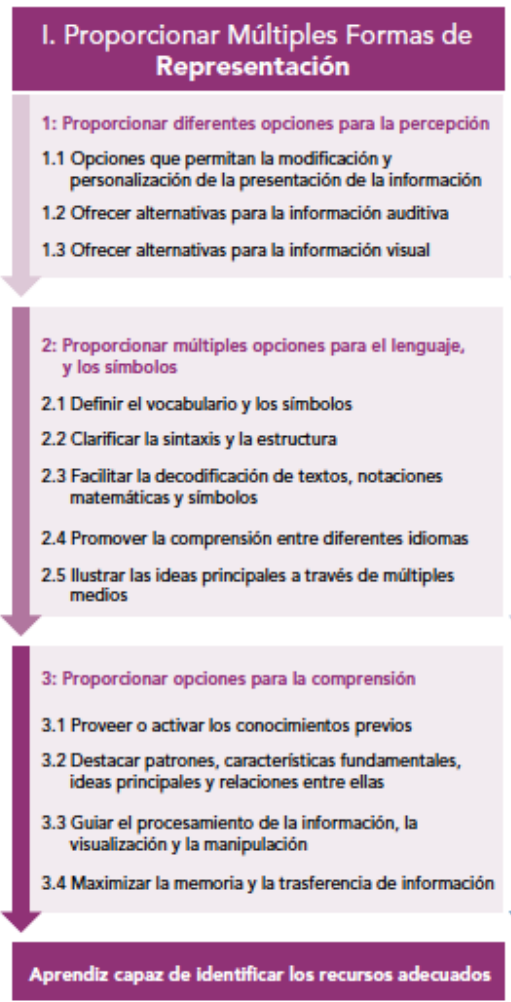

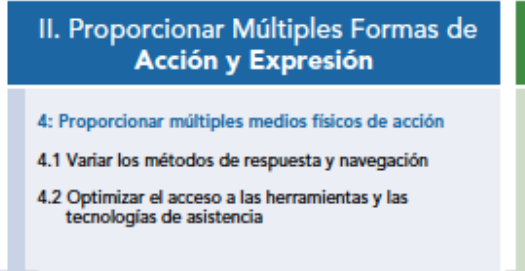

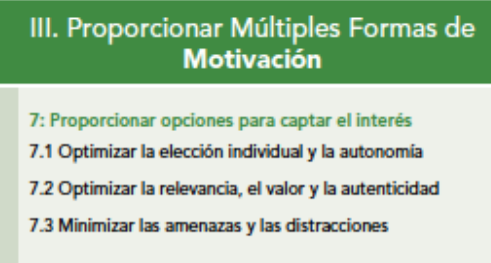

5: Proporcionar opciones para la expresión y la

1 Usar múltiples opciones de medios de comunicación

5.2 Usar mültiples herramientas para la construcción y la composición

5.3 Construir fluidez de aprendizaje con niveles

graduados de apoyo para la práctica y la ejecución
8: Proporcionar opciones para mantener el esfuerzo

y la persistencia

8.1 Resaltar la relevancia de las metas y los objetivos

8.2 Variar los niveles de desafio y apoyo

8.3 Fomentar la colaboración y la comunidad

8.4 Incrementar el dominio de retroalimentación orientada
6: Proporcionar opciones para las funciones ejecutivas

6.1 Guiar el establecimiento de metas adecuadas

6.2 Apoyar la planificación y el desarrollo de estrategias

6.3 Facilitar la gestión de información y de recursos

6.4 Aumentar la capacidad para monitorear el progreso
: Proporcionar opciones para la auto-regulación

Promover expectativas y creencias que optimicen la motivación

2 Facilitar niveles graduados de apoyo para copiar habilidades y estrategias

9.3 Desarrollar la auto-evaluación y la reflexión

Figura 1. Pautas 2.o sobre el Diseño Universal para el Aprendizaje (NCUDL, 2012).

Complementando el desarrollo de currículos basados en DUA, un segundo eje principal de trabajo es el uso de las TIC como medio para favorecer la flexibilidad en los contenidos, los métodos y la evaluación (Asunción, 2008; Burgstahler, 2008). Los profesores, que de manera regular, proporcionan oportunidades a sus estudiantes de usar la tecnología de manera autosuficiente, consiguen convertir a sus estudiantes en aprendices más efectivos (Hitchcock y Stahl, 2003). Incluso aquellos profesores que incorporan tecnología en la práctica educativa, si en sus clases existen estudiantes con las suficientes habilidades tecnológicas, consiguen que los alumnos logren los objetivos de aprendizaje de manera más independiente (Grabe y Grabe, 2004). En un estudio descrito por Arter, Perlis, Ruthkosky, Burkhouse, y Holmes (2008), se demuestra que la participación y la motivación por parte de los estudiantes aumenta en más del $50 \%$ cuando la tecnología se utiliza adecuadamente y siguiendo los principios del DUA.

De especial importancia en la formación continua del docente para atender a la diversidad, es conocer ciertos principios de accesibilidad en el uso de las TIC, para que éstas puedan ser utilizados por todos los estudiantes (Coyne, Pisha, Dalton, Zeph y Smith, 2013), independientemente de cuáles sean sus características y circunstancias personales. En una revisión de las barreas en el uso de las TIC en clase (Bingimlas, 2009) se describe las competencias relacionadas con los conocimientos de los profesores sobre la accesibilidad en entornos informáticos como críticas, y una de las principales barreras en el acceso a la información por parte de los estudiantes. 


\section{OBJETIVO}

El objetivo principal de este trabajo es presentar una experiencia llevada a cabo en España, sobre cómo atender a la diversidad en el aula siguiendo los principios del DUA y el uso equitativo de las TIC.

Para lograr dicho objetivo, este trabajo ha tenido en cuenta los cursos de formación continua que se imparten en los centros de recursos del profesorado.

\section{DESARROLLO}

Esta experiencia, llevada a cabo durante varios cursos académicos, ha consistido en dar a conocer a profesores de educación Primaria y Secundaria los beneficios del uso adecuado de las TIC teniendo en cuenta la diversidad del aula siguiendo los principios del diseño universal para el aprendizaje.

La importancia de un uso responsable de las TIC, debe tener asociado unos conocimientos sobre los posibles tipos de alumnos que se pueden encontrar en las clases de hoy en día, así como habilidades informáticas que tengan en cuenta criterios de accesibilidad informática y ofimática. Además, estas competencias deben centrarse en cómo usan las personas con discapacidad los recursos TIC, cuáles son sus principales características y cuál es el modo en el que los estudiantes con discapacidad se enfrentan al uso de las mismas.

A lo largo de los dos últimos cursos académicos, se han realizado diversos seminarios en los Centros de Recursos del Profesorado de diferentes ciudades de España. En estas acciones formativas han participado alrededor de unos 50 profesores, tanto de Educación Primaria, como Educación Secundaria. El perfil de los participantes ha sido de maestros y maestras de educación especial, así como orientadores y tutores de los ciclos finales de Primaria.

Los seminarios se han llevado a cabo en varias sesiones en días consecutivos, en las que la duración aproximada de cada sesión ha sido de entre tres y cinco horas, siendo el total del seminario de 10 horas.

El contenido de estas formaciones tiene su base en los conocimientos necesarios sobre la discapacidad, el paradigma del diseño universal y su aplicación al ámbito educativo, y el uso de las TIC desde una perspectiva de atención a la diversidad siguiendo los principios del DUA. El siguiente cuadro muestra los diferentes módulos de los que se ha compuesto el seminario.

Tabla 2. Estructura de curso de formación sobre el uso de las TIC y diseño universal para el aprendizaje.

\begin{tabular}{ccc}
\hline Módulo & \multicolumn{1}{c}{ Contenido } \\
\hline Módulo 1 & $\bullet$ & $\begin{array}{l}\text { Conceptos claves sobre discapacidad } \\
\text { Uso TIC en función de la discapacidad }\end{array}$ \\
\hline Módulo 2 & $\bullet$ & Introducción al Diseño Universal \\
\hline
\end{tabular}




\begin{tabular}{lll}
\hline & Diseño Universal para el Aprendizaje \\
& Pautas del Diseño Universal para el Aprendizaje y el uso de las TIC \\
& siguiendo sus principios \\
\hline Módulo 3 & $\begin{array}{l}\text { Accesibilidad informática } \\
\text { - }\end{array}$ \\
& $\begin{array}{l}\text { Accesibilidad ofimática (Procesadores de textos, programas de } \\
\text { presentación de diapositivas, PDF, etc.) }\end{array}$ \\
\hline
\end{tabular}

De este modo, el módulo 1, pretende que los asistentes conozcan la discapacidad desde un punto de vista de usuario de las TIC, y cómo éstas pueden ser una ayuda muy importante debido a sus características, su flexibilidad en el uso, su versatilidad temporal para el consumo de información, y las posibilidades de adaptación en base a las características individuales. Pero, a su vez, este módulo está encaminado a la identificación de las posibles barreras que el uso de las TIC puede suponer para una persona con discapacidad si no se ha programado un uso correcto por parte del profesor. Se hace un recorrido por las principales discapacidades y cuáles son los principales problemas que se pueden encontrar los alumnos con discapacidad en el uso de ciertas tecnologías en función de su discapacidad.

En el módulo 2, se presenta el paradigma del diseño universal y cómo este enfoque ha sido aplicado a la educación para atender a la diversidad. De manera más concreta, se desarrolla el concepto de diseño universal para el aprendizaje y se describen sus tres principios. Además, se presentan las pautas sobre DUA que avalan su aplicación en la educación básica. Finalmente, dentro de este segundo módulo, se explican algunas de las principales herramientas informáticas que se usan en la actualidad en los actos educativos (plataforma de aprendizaje virtual, herramientas colaborativas, redes sociales educativas, etc.) desde una óptica basada en la diversidad y en la aplicación de los principios del DUA.

Por último, el módulo 3, está centrado en describir las principales habilidades ofimáticas relacionadas con la accesibilidad de las herramientas más comunes de uso en las aulas. Se explica cómo realizar documentos accesibles en procesadores de texto, programas de presentación de diapositivas, crear PDF accesibles, etc. Además, se presentan los beneficios que supone el crear documentos accesibles desde el principio con un carácter proactivo.

Los resultados de los cuestionarios de satisfacción de los participantes, muestran que el interés por la temática y los contenidos fue de más de ocho puntos sobre una escala de diez. Igualmente, su aplicabilidad en el aula estuvo cercana a ocho, así como el desarrollo de competencias por parte de los asistentes. La nota global de la actividad se situó en más de 8.25 y la satisfacción de la actividad realizada fue de 8.10 .

Finalmente, a través de una metodología de carácter más cualitativo, en base a la creación de grupos de discusión sobre la viabilidad de los contenidos expuestos, de la situación de las personas con necesidades educativas especiales y la formación que los actuales maestros y profesores de secundaria tienen en la utilización de recursos TIC, los profesores manifestaron la necesidad de avanzar en el conocimiento de estos nuevos enfoques que favorecen su formación en aspectos relacionados con las TIC y metodologías que favorecen la atención a la diversidad en el aula. También, se expresaron las inquietudes que desde el colectivo de 
educadores se plantean cuando en el aula hay chicos y chicas con discapacidad y las ayudas que provienen del exterior no siempre llegan a tiempo. Otra preocupación expresada fue la utilización de las TIC por parte del alumnado y es que en la actualidad son muchos los casos en los que el alumnado tiene más conocimiento del uso de las TIC que el propio profesorado.

\section{CONCLUSIONES Y DISCUSIÓN}

Ciertas investigaciones a nivel internacional demuestran que el entrenamiento, aún en breves períodos de tiempo, sobre temas relacionados con el uso de las TIC y la aplicación del paradigma del DUA, favorecen las percepciones de los propios estudiantes sobre su aprendizaje (Duk y Murillo, 2013; Engleman y Schmidt, 2007; y Spooner et al., 2007).

Los resultados de las evaluaciones de la experiencia aquí presentada, también describen como algo positivo y necesario el conocer este tipo de enfoques para atender a la diversidad, además de los conceptos relacionados con el uso de las TIC según las necesidades individuales.

Por lo tanto, parece razonable afirmar que se debería tener en cuenta en las programaciones de los centros de formación del profesorado, las necesidades que demandan los profesores y que favorecen la participación de todos los estudiantes en su proceso educativo. Y, aunque ésta es solo una de las vías para lograr una educación lo más inclusiva posible, en la actualidad, es un medio fundamental para que los maestros y profesores que ya están en activo puedan disponer de las herramientas necesarias, tanto a nivel informático como a nivel de conocimientos sobre la diversidad en el aula.

Finalmente, cabe destacar, que el paradigma del diseño universal para el aprendizaje es un enfoque que cada día tiene más auge y que debe ir integrándose de manera progresiva en las políticas educativas de los países desarrollados o en vías de desarrollo. Además, una última vía de expansión de este tipo de enfoques, es a través de la inclusión de competencias relacionadas con estos temas en la formación universitaria que reciben los futuros profesores que estarán en las aulas a lo largo de los próximos años. Igualmente, los conocimientos tecnológicos que se requieren para atender a la diversidad en el aula, son una tarea pendiente de muchos profesores que no comenzaron su etapa profesional con la integración de las TIC en el aula, y que hoy en día, no han tenido más remedio que adaptarse a las necesidades de la sociedad del Siglo XXI.

\section{REFERENCIAS}

Ainscow, M. (2006). From special education to effective schools for all: a review of progress so far. En The Sage Handbook of Special Education, L. Floiran, (pp. 146-159). London: Sage.

Armstrong, F. \& Moore, M. (2004). Action Research for Inclusive Education: Changing Places, Changing Practices, Changing Minds. London: Routledge Falmer. 
Arter, P., Perlis, S., Ruthkosky, K., Burkhouse, E. \& Holmes, J. (2008). Using Student Response Systems for Curricular Redesign en Education Courses. In Proceedings of Society for Information Technology y Teacher Education International Conference 2008 (pp. 5203-5208). Chesapeake, VA. Recuperado de http://www.editlib.org/p/28102

Asuncion, J. (2008). Computer technologies and postsecondary students with disabilities: Implications of recent research for rehabilitation psychologists. Rehabilitation Psychology, 48(3), 207-214. Recuperado de http://dc160.dawsoncollege.qc.ca/pubs/abComputer_Technologies_and_Postse condaryFT.pdf

Bingimals, K. A. (2009). Barriers to Successful Integration of ICT en Teaching and Learning Environments. A Review of the Literature. Eurasia Journal of Mathematics, Science and Technology Education, 5(3), 235-245. Recuperado de http://www.ejmste.org/v5n3/EURASIA_v5n3_Bingimlas.pdf

Booth, T. \& Ainscow, M. (2002). Index for Inclusion. Developing learning and participation in schools. CSIE Mark Vaughan.

Burgstahler, S. (2008). Universal Design of Technological Enviroments: From Principles to Practices. En Universal Design in Higher Education. From principles to practices. Cambirdge, MA.: Harvard Education Press.

Coyne, P., Pisha, B., Dalton, B., Zeph, L. A. \& Smith, N. (2013). Literacy by Design: A Universal Design for Learning Approach for Students With Significant Intellectual Disabilities. Remedial and Special Education, 33(3), 162-172. doi:10.1177/0741932510381651 Recuperado de http://rse.sagepub.com/content/early/2010/08/30/0741932510381651.abstract

Díez, E., Alonso, A., Verdugo, M. A., Campo, I., Sancho, I., Sánchez, S.,... Moral, E. (2011). Espacio Europeo de Educación Superior: estándares e indicadores de buenas prácticas para la atención a estudiantes universitarios con discapacidad Salamanca: Instituto de Integración en la Comunidad, Universidad de Salamanca. Recuperado de: http://sid.usal.es/libros/discapacidad/26032/81/espacio-europeo-de-educacion-superior-estandares-e-indicadores-de-buenaspracticas-para-la-atencion-de-estudiantes-universitarios-con-discapacidad.aspx

Duk, C., y Murillo, J. (2013). El valor del "Diseño Universal de Aprendizaje" para la educación inclusiva. Revista Latinoamericana de Educación Inclusiva, 7(1), 7-9. Recuperado de http://www.rinace.net/rlei/numeros/vol7-num1/Editorial.pdf

Dyson, A., Howes, A. \& Roberts, B. (2002). A systematic review of the effectiveness of school-level actions form promoting participation by all students. London: EPPI-Centre, Social Science Research Unit, Institute of Education.

Engleman, M. \& Schmidt, M. (2007). Testing an experimental universally designed learning unit in a graduate level online teacher education course. Journal of Online Learning and Teaching, 3(2), 112-132. 
Grabe, M. \& Grabe, C. (2004). Integrating technology for meaningful learning. New York: Houghton Miffllin.

Hitchcock, C. \& Stahl, S. (2003). Assistive Technology, Universal Design, Universal Design for Learning: Improved learning opportunities. Journal of Special Educational Technology, 19(4), 45-52.

McGuire, J., Scott, S. \& Shaw, S. (2006). Universal Design and Its Aplications in Educational Environments. Remedial and Special Education, 27(3), 166-175. Recuperado de http://www.regent.edu/acad/schedu/pdfs/UD_Article_2006.pdf

Meyer, A. \& Rose, D. (2009). A Policy Reader in Universal Design for Learning (Gordon, D., Gravel, J. y Schifer, L.). Cambridge, MA: Harvard Education Press.

National Center for Universal Design for Learning. (2012). UDL Guideline - Version 2.0. Recuperado de http://www.udlcenter.org/aboutudl/udlguidelines

Ribeiro, J. y Sánchez, S. (2013). Inclusión Educativa a través de las TIC. Indagatio Didactica, 5(4), 147-160.

Rose, D. \& Meyer, A. (2000). Universal Design for Learning. Journal of Special Education Technology, 15, 67-70.

Rose, D. \& Meyer, A. (2002). Teaching Every Student in the Digital Age: Universal Design for Learning. VA: ASCD.

Rose, D. \& Meyer, A. (2006). A Practical Reader in Universal Design for Learning. Harvard Education Press.

Rose, D., Meyer, A. \& Hitchcock, C. (2005). The Universally Designed Classroom. Harvard Education Press.

Ruíz, R., Solé, L., Echeita, G., Sala, I., y Datsira, M. (2012). El principio del "Universal Design". Concepto y desarrollos en la enseñanza superior. Revista de Educación. doi:10-4438/1988-592X-RE-2010-359-100. Recuperado de http://www.revistaeducacion.educacion.es/doi/359_100.pdf

Spooner, F., Baker, J., Harris, A., Ahlgrim-Delzell, L., y Browder, D. (2007). Effects of Training in Universal Design for Learning on Lesson Plan Development. Remedial and Special Education, 28(2), 108-116. 